\title{
DESIGN, FABRICATION AND OPERATION OF A HOT ELECTRON RESONANT TUNNELING TRANSISTOR
}

\author{
U.K. Reddy, I. Mehdi, R. K. Mains and G. I. Haddad \\ Center for High-Frequency Microelectronics \\ Department of Electrical Engineering and Computer Science \\ The University of Michigan \\ Ann Arbor, MI 48109-2122
}

\begin{abstract}
Transistors employing resonant tunneling injection of hot electrons into a thin quantum well base region have been fabricated. The base region in these transistors is formed by a narrow bandgap material like InGaAs so that the first level is a confined one lying below the Fermi level in the contact regions. This results in charge transfer into the bound state in the quantum well thus allowing independent control of the base electrostatic potential. Theoretical calculations showing the importance of various device parameters in the design of a resonant tunneling transistor are presented and preliminary results showing the capability of transistor action in such devices are presented.
\end{abstract}

\section{KEYWORDS}

Resonant tunneling; hot electrons; bound state; quantum well base; transistor action.

\section{INTRODUCTION}

The use of the resonant tunneling phenomenon for three terminal devices is interesting because of the possibility of realizing extremely high speed devices. Two possible means of utilizing resonant tunneling structures (RTSs) have been under investigation. The first approach involves using the conventional RTS in connection with existing three terminal devices such as FETs and BJTs. The RTS can be placed in the emitter or base or in series with the gate and interesting results have been demonstrated (Capasso and co-workers, 1988; Woodward and co-workers, 1988). The RTS can also be incorporated into hot electron transistors where again encouraging results have been obtained (Yokoyama and co-workers, 1988). The second approach involves contacting the quantum well directly and using the well contact as the control electrode. This approach has been suggested as early as 1963 (Davis and Hosak, 1963). A necessary modification of this approach is to use a modified RTS where charge is transferred

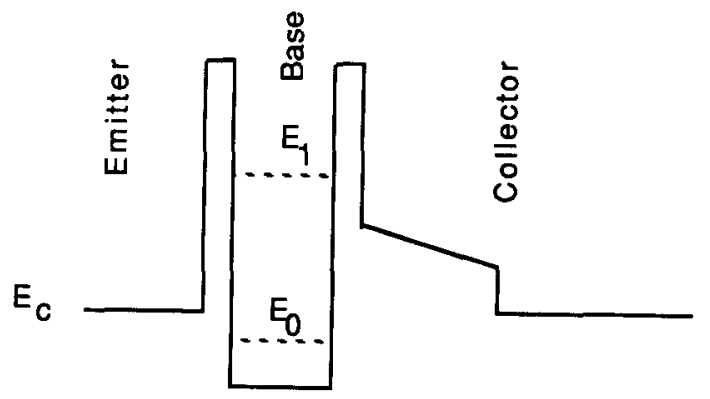

Fig. 1. Schematic conduction band diagram of the resonant tunneling transistor showing the bound state $E_{0}$ in the deep quantum well (base). Electrons from the emitter region tunnel resonantly through the first excited state $E_{1}$ and are collected in the collector. Electrons from the heavily doped GaAs regions populate the lower lying bound state $\mathrm{E}_{0}$ in the quantum well making the base conducting. 
into a deep quantum well, enabling formation of a low resistance base contact, and thus by applying a voltage on the base the collector current can be modulated. A theoretical analysis of this approach has been presented (Haddad and co-workers, 1989; Schulman and Waldner, 1988). Experimental results involving this approach have been reported by Reed and co-workers (1989) in a bipolar device while preliminary unipolar device results have been presented by Reddy and co-workers (1989). In this paper a self consistent quantum mechanical simulation is used to study the effect of doping and spacer layer thickness on the ability of transferring charge into the quantum well. These results are then used to design a transistor. The transistor structure has been fabricated and both two and three terminal I-V characteristics have been measured.

The transistor structure is a modified double barrier resonant tunneling structure. The distinguishing features of this structure are a deep quantum well with a bound state and an extended collector barrier as shown in Fig. 1. The bound state is populated by transfer of charge from the contacting regions, thus making the quantum well a conducting region. The extended barrier prevents the leakage of charge from the bound state in the base region into the collector when the collector is biased. The operation of the transistor relies on the injection of hot electrons through the first excited state in the quantum well base. These electrons have large energies compared to the bound state electrons and the conduction mechanism is by resonant tunneling. A detailed description has been presented (Haddad and co-workers, 1989).

\section{THEORETICAL FORMALISM AND RESULTS}

The operation of the RTT strongly depends on the existence of the bound state in the deep quantum well. To study the effect of device parameters on the existence and behavior of the bound state a quantum mechanical simulation has been developed. The self-consistent simulation takes into account the charge in the well. The contact regions outside the double-barrier structure are treated using a Thomas-Fermi equilibrium model, i.e. constant Fermi
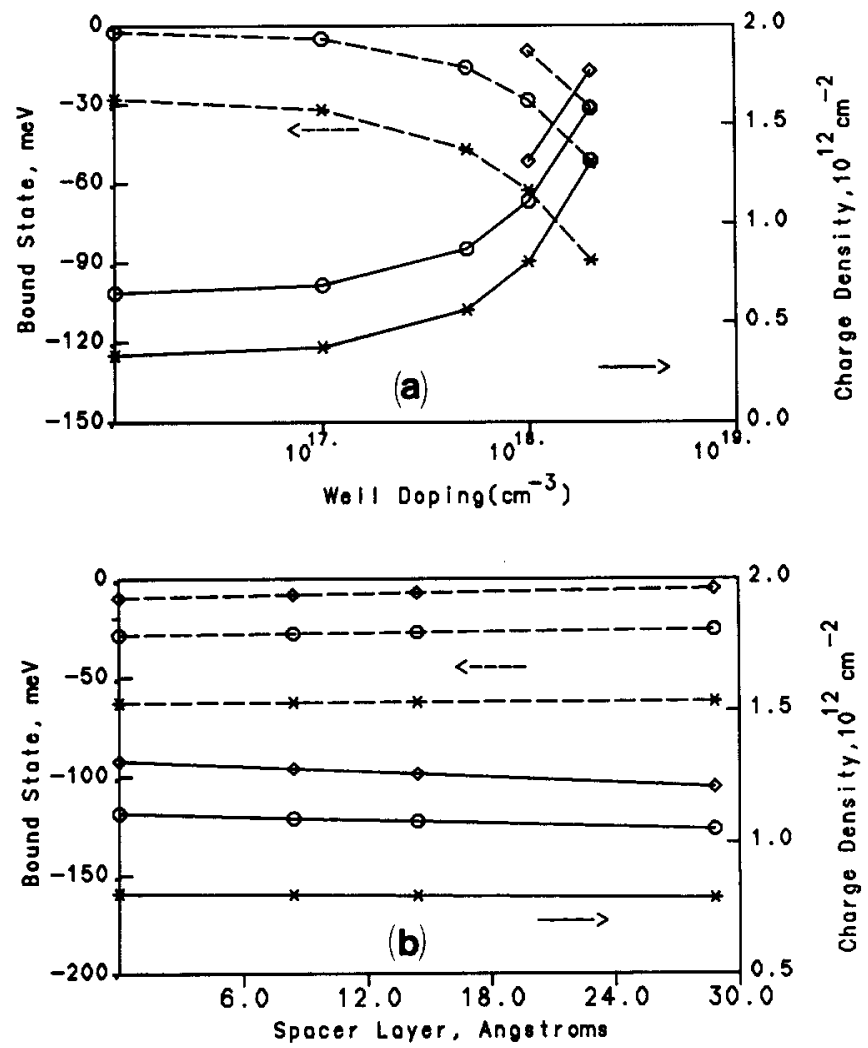

Fig. 2 Variation of the bound state position and bound state charge as a function of (a) well doping, (b) spacer layer thickness. The various curves represent different emitter and collector doping levels: $\diamond=1 \times 10^{18} \mathrm{~cm}^{-3}, 0=5 \times$ $10^{17} \mathrm{~cm}^{-3}$, and $x=1 \times 10^{17} \mathrm{~cm}^{-3}$. For (a) $0 \AA$ spacer layers were used while the results in (b) assume a well doping of $1 \times 10^{18} \mathrm{~cm}^{-3}$. 
levels within each region and equilibrium Fermi-Dirac statistics are assumed. Within the double-barrier structure, electron concentrations are obtained from the time-independent Schrödinger equation. The concentrations obtained inside the device are then self-consistently coupled to the Thomas-Fermi model through matching conditions connecting the two contact regions.

In order to solve for the bound state the energy from the bottom of the well is scanned and a finite difference form of Schrödinger's equation is solved until a well confined wave function is obtained. The corresponding wavefunction is normalized to unity and then multiplied by the two dimensional density of states and occupation probability function to obtain the charge density in the well.

It is found that parameters such as barrier height and barrier width though important for peak-to-valley ratio and current density do not affect the charge in the bound state dramatically. Figure 2(a) shows the variation in the position of the bound state and the charge in the bound state as a function of doping in the well and the emitter (emitter and collector dopings are assumed to be the same). Figure 2(b) shows the same information for varying spacer layer widths. These figures are for zero bias. The position of the bound state is specified with reference to the band edge outside the barriers which is taken to be zero. Thus a larger negative bound state energy would imply that the bound state is deeper in the quantum well. For these results the barrier width was $40 \AA$, barrier height $1.0 \mathrm{eV}$, well depth $0.19 \mathrm{eV}$, well width $80 \AA$, electron mass in the emitter and collector $0.067 m_{o}$, electron mass in the well, $0.056 m_{o}$, electron mass in the barriers $0.15 m_{o}$, and the temperature was $300 \mathrm{~K}$. The extended barrier was $500 \AA$ long starting with $\mathrm{Al}_{0.20} \mathrm{Ga}_{0.80} \mathrm{As}$ and ending with $\mathrm{Al}_{0.10} \mathrm{Ga}_{0.90} \mathrm{As}$ on the collector side. $\mathrm{A}$ linear extrapolation was used to calculate the effective electron mass in the extended barrier. These material parameters correspond to the structure shown in Fig. 3.

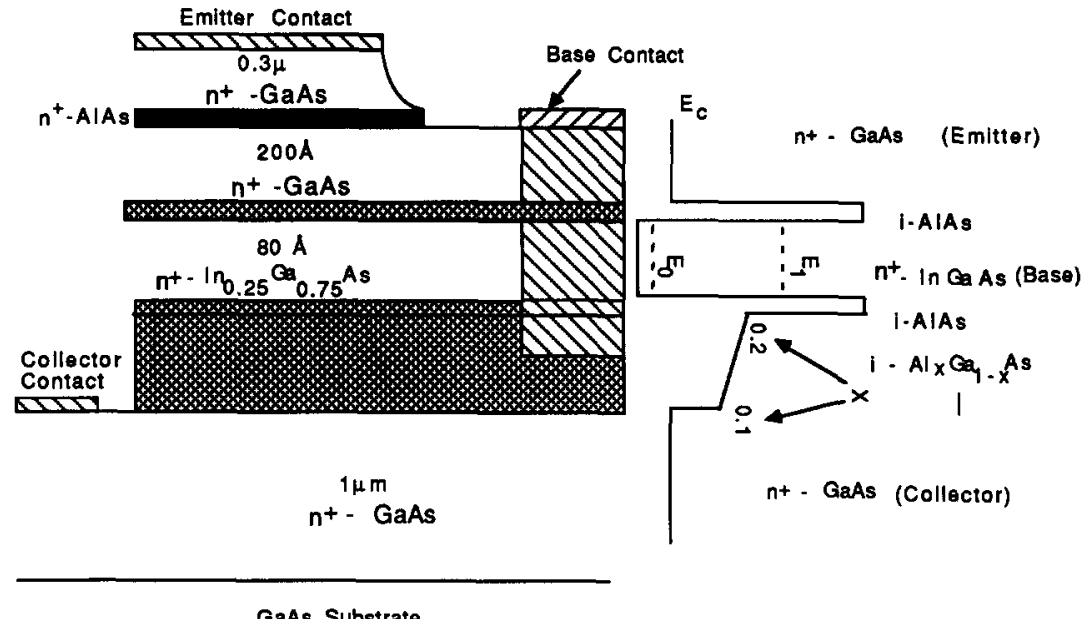

Fig. 3 Cross sectional view of the RTT and its conduction band diagram. The base ohmic is diffused into the base (quantum well) region to contact the two-dimensional electron gas in the bound state without shorting the collector region.

From Fig. 2(a) it is seen that as the well doping is increased the charge in the bound state increases for a constant emitter doping. Also for a constant well doping the charge in the bound state increases with increasing emitter doping. Since for a low base resistance it is desirable to have a large number of carriers in the well, both the well and emitter should be doped heavily. For the transistor structure that has been fabricated the emitter and well were both doped to $1 \times 10^{18} \mathrm{~cm}^{-3}$. Using the above described simulation this would imply that the bound state energy is only a few $\mathrm{meV}$ from the band edge and the charge in the bound state is $2 \times 10^{12} \mathrm{~cm}^{-2}$. It is, however, important to note that our calculation of the bound state energy does not take into account the exchange interaction effect. This effect has been shown (Bandara and co-workers, 1988) to lower the ground state subband energies in doped quantum wells.

Figure 2(b) shows that for a well doping of $1 \times 10^{18} \mathrm{~cm}^{-3}$ spacer layer thickness between 0 and $30 \mathrm{~A}$ does not strongly affect the bound state energy. The bound state energy and the charge in it, of course, do depend on the 
emitter doping. For the fabricated structure a spacer layer of $10 \AA$ was used primarily to prevent dopant diffusion into the barriers.

\section{EXPITAXIAL GROWTH AND DEVICE FABRICATION}

The eptiaxial layers were grown by molecular beam epitaxy (MBE) on semi-insulating GaAs substrates at $600^{\circ} \mathrm{C}$. First, a five period GaAs/AlAs superlattice was grown followed by a 1 micron thick $\mathrm{n}^{+}$GaAs layer. The double barrier was grown on top of the $500 \AA$ graded $\mathrm{Al}_{x} \mathrm{Ga}_{1-x} \mathrm{As}$ barrier. The double barrier structure consists of $80 \AA n^{+}$-InGaAs quantum well bounded on either side by $40 \AA$ thick AlAs barrier layers. This was followed by a $200 \AA n^{+}$-GaAs and a heavily doped $50 \AA$ AlAs layer and finally a 0.3 micron thick $n^{+}$GaAs layer was grown on the double barrier structure. The heavily doped AlAs layer serves as an etch stop during device fabrication. The $200 \AA \mathrm{n}^{+}$-GaAs layer between the heavily doped AlAs and first AlAs barrier is completely depleted by surface Fermi pinning and therefore, does not short the emitter mesa and base contact.

The structures were fabricated into three terminal devices as shown in Fig. 3 using standard photolithography techniques, with wet chemical etching for mesa formation and lift-off process for ohmic metalization. The chemical etching was done by $\mathrm{NH}_{4} \mathrm{OH}: \mathrm{H}_{2} \mathrm{O}_{2}: \mathrm{H}_{2} \mathrm{O}(1: 1: 100)$ with an etch rate of $33 \AA / \mathrm{s}$. The multilayer ohmic metalization consisted of $\mathrm{Ni} / \mathrm{Ge} / \mathrm{Au} / \mathrm{Ti} / \mathrm{Au}$ followed by annealing at 450 degrees for 60 seconds in flowing nitrogen. The quantum well base was contacted by electrically monitoring the etching process.

\section{EXPERIMENTAL RESULTS AND DISCUSSION}

Both two terminal and three terminal DC I-V characteristics of the transistors were measured and the results are shown in Figs. 4 and 5 . When a voltage is applied between the emitter and collector with base terminal open, diode like behavior showing negative differential resistance (NDR) is observed. At room temperature the current peak-to-valley ratio is very small but clearly observable. The two terminal I-V plot at $77 \mathrm{~K}$ is shown in Fig. 4. NDR is seen in only one direction since the double barrier structure is asymmetric. The low peak-to-valley ratio observed is probably due to electron scattering from the bound state charge.

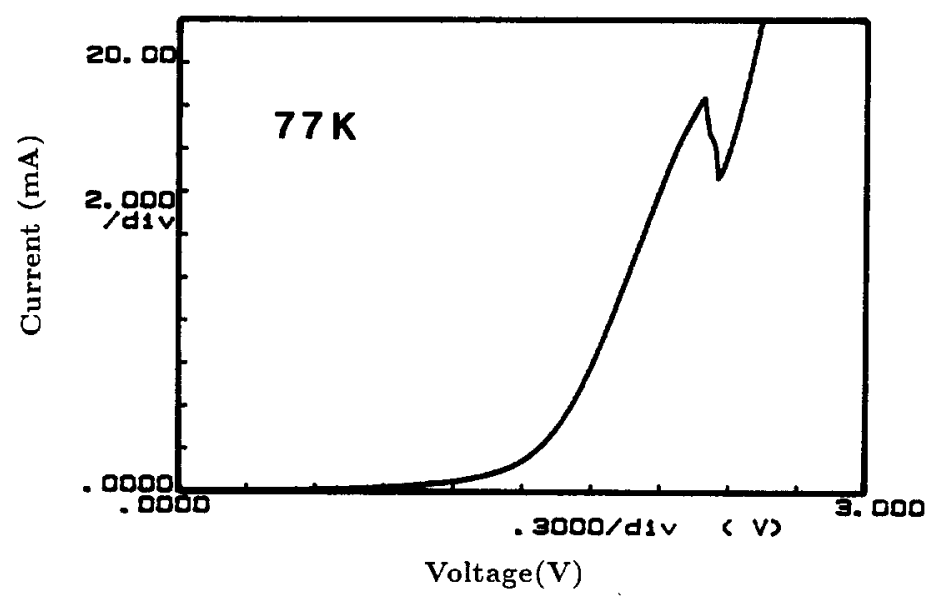

Fig. 4 Two terminal I-V characteristic for the fabricated devices at $77 \mathrm{~K}$. The applied voltage is between the collector and emitter with base open. The measurements were done with the emitter grounded and the collector biased positively.

The common-emitter characteristics of the RTT at room temperature are shown in Fig. 5 for different base voltages. It is seen that the collector current is modulated by the base voltage. However, the device showed very low gain and the collector current shows no saturation for a given base voltage. The degradation in gain, we believe, is due to Fermi pinning in the region between the base contact and the active base region. This results in a large voltage drop across the extrinsic base region. Further, the Fermi pinning in the extrinsic base draws charge from the bound state in the quantum well base, making the base less conducting. Thus, the emitter is no longer shielded electrically from the potential on the collector terminal resulting in the observed lack of collector 
current saturation. To overcome this problem of surface Fermi pinning a self-aligned process is under development. Once this problem is solved not only is the gain expected to improve but this device could act as a prototype

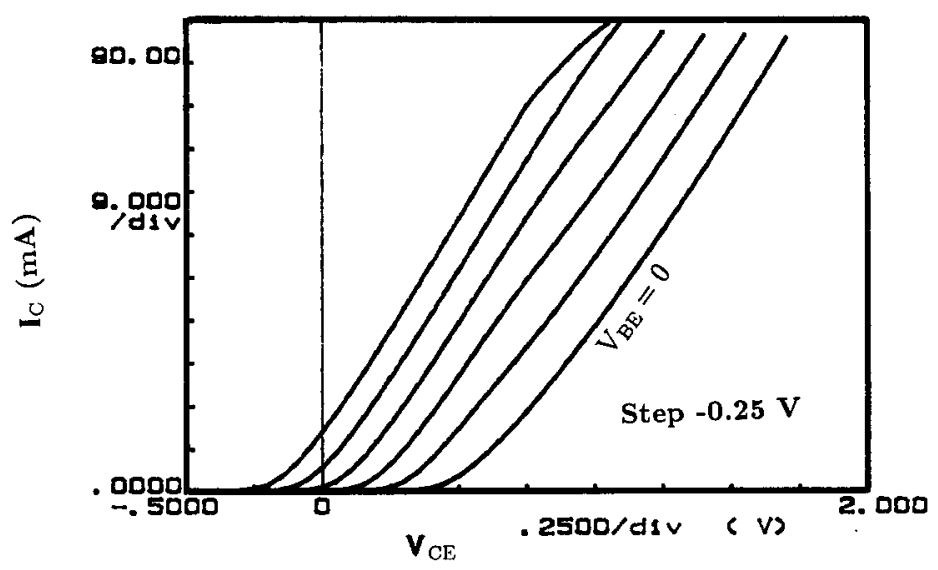

Fig. 5 Common-emitter characteristics of the RTT for different base voltages.

in understanding hot electron transport in ultra small structures, unipolar transistor action, and physics of two dimensional systems.

\section{CONCLUSION}

Based on a design using a self consistent quantum mechanical simulation resonant tunneling transistors have been fabricated by contacting the quantum well. The base region is made conducting by using a smaller bad gap material so that a bound state lying below the GaAs band edge is formed in the quantum well. Devices exhibit negative differential resistance when operated as two terminal diodes at room temperature and $77 \mathrm{~K}$. The transistors show low gain due to surface depletion in the extrinsic base region. A self-aligned process for overcoming this problem is under development.

\section{ACKNOWLEDGEMENT}

We thank J. Oh, J. Pamulapati, and P. K. Bhattacharya for material growth. This work was supported by the U. S. Army Research Office, contract no. DAAL03-87-K-0007.

\section{REFERENCES}

Bandara, K.M.S.V., Coon, D. D., Byungsung, O., Lin, Y. F., and Francombe, M. H. (1988). Exchange interactions in quantum well subbands. Appl. Phys. Lett, $\underline{53}, 1931-1933$.

Capasso, F., Sen, S., Cho, A. Y., Gossard, A. C., and Spah, R. J. (1988). Resonant tunneling through quantum wells: physics and device applications. Solid-Sate Electronics, 31, 723-729.

Davis, R. H., and Hosak, H. H. (1963), Double barrier in thin film triodes. J. Appl. Phys., 34, 864-868.

Haddad, G. I., Mains, R. K., Reddy, U. K. and East, J. R. (1989). A proposed narrow-band-gap base transistor structure. Superlattices and Microstructures, 5, 437-442.

Reddy, U. K., Haddad, G. I., Mehdi, I., and Mains, R. K. (1989). Presented at the 4th Intl. Symposium on Nanostructure Physics, College Station, TX, March 15-19, 1989.

Reed, M. A., Frensley, W. R., Matyi, R. J., Randall, J. N., and Seabaugh, A. C. (1989). Realization of a three-terminal resonant tunneling device: the bipolar quantum resonant tunneling transistor. Appl. Phys. Lett., 54, 1034-1036.

Schulman, J. N., and Waldner, M. (1988). Analysis of second level resonant tunneling diodes and transistors. J. Appl. Phys., 63, 2859-2861.

Woodward, T.K., McGill, T. C. , Burnham, R. D., and Chung, H. F. (1989). Resonant tunneling field-effect transistors. Superlattices and Microstructures, 4, 1-9.

Yokoyama, N., Imamura, K., Ohnishi, H., Mori, T., Muto, S., and Shibatoni, A. (1988). Resonant-tunneling hot electron transistors (RHET). Solid-State Electronics, 31, 577-582. 